\title{
Identification of prognosis-related genes and construction of multi-regulatory networks in pancreatic cancer microenvironment by bioinformatics analysis
}

\author{
Tong Li®D, Qiaofei Liu, Ronghua Zhang, Quan Liao* ${ }^{*}$ and Yupei Zhao*
}

\begin{abstract}
Background: As one of the most lethal cancers, pancreatic cancer has been characterized by abundant supportive tumor-stromal cell microenvironment. Although the advent of tumor-targeted immune checkpoint blockers has brought light to patients with other cancers, its clinical efficacy in pancreatic cancer has been greatly limited due to the protective stroma. Thus, it is urgent to find potential new targets and establish multi-regulatory networks to predict patient prognosis and improve treatment.

Methods: We followed a strategy based on mining the Cancer Genome Atlas (TCGA) database and ESTIMATE algorithm to obtain the immune scores and stromal scores. Differentially expressed genes (DEGs) associated with poor overall survival of pancreatic cancer were screened from a TCGA cohort. By comparing global gene expression with high vs. Iow immune scores and subsequent Kaplan-Meier analysis, DEGs that significantly correlated with poor overall survival of pancreatic cancer in TCGA cohort were extracted. After constructing the protein-protein interaction network using STRING and limiting the genes within the above DEGs, we utilized RAID 2.0, TRRUST v2 database and degree and betweenness analysis to obtain non-coding RNA (ncRNA)-pivotal nodes and transcription factor (TF)pivotal nodes. Finally, multi-regulatory networks have been constructed and pivotal drugs with potential benefit for pancreatic cancer patients were obtained by screening in the DrugBank.
\end{abstract}

Results: In this study, we obtained 246 DEGs that significantly correlated with poor overall survival of pancreatic cancer in the TCGA cohort. With the advent of $38 \mathrm{ncRNA}$-pivotal nodes and 7 TF-pivotal nodes, the multi-factor regulatory networks were constructed based on the above pivotal nodes. Prognosis-related genes and factors such as HCAR3, PPY, RFWD2, WSPAR and Amcinonide were screened and investigated.

Conclusion: The multi-regulatory networks constructed in this study are not only beneficial to improve treatment and evaluate patient prognosis with pancreatic cancer, but also favorable for implementing early diagnosis and personalized treatment. It is suggested that these factors may play an essential role in the progression of pancreatic cancer.

Keywords: TCGA, Differentially expressed gene, Multi-regulatory network, Pancreatic cancer, Tumor microenvironment

*Correspondence: lqpumc@126.com; zhao8028@263.net Department of General Surgery, Peking Union Medical College Hospital, Chinese Academy of Medical Sciences \& Peking Union Medical College, Beijing 100730, China

\section{Background}

Pancreatic cancer currently is one of the top three leading causes of cancer-related death in the United States and its death toll is rising dramatically [1]. The only chance 
of cure for pancreatic cancer patients is surgical resection [2]. For decades, despite the concerted efforts, the five-year survival rate remains dismal for all stages combined [1]. The lack of early detection tests and recognizable symptoms or signs results in late diagnoses [3]. The advent of various systemic therapies has just provided limited efficacy for pancreatic cancer patients to date [4, $5]$, which may be due to the abundance of tumor stromal content $[6,7]$.

The pancreatic cancer stroma, which has aptly been termed the tumor microenvironment (TME), is the cellular milieu where the tumor is located. The stroma occupies the majority of the tumor mass and is heterogeneously comprised of dynamic assortment of nonneoplastic cells and extracellular matrix components, including fibroblastic, vascular and immune cells, cytokines and growth factors $[8,9]$. The influences of the stroma in pancreatic cancer are as manifold as its components [7]. In the pancreatic TME, immune cells and stromal cells are the two main types of non-neoplastic components and have been proposed to be of value for the diagnosis and prognosis of tumors [10-13].

The comprehensive understanding of immune cells and stromal cells in pancreatic cancer tissues may shed light on the tumor pathophysiology and help with developing wholesome prognostic and predictive models $[8$, 14]. Thus, it is important to evaluate immune scoring and matrix scoring to investigate the composition of stromal cells and immune cells and evaluate tumor purity $[12,14$, 15]. Algorithms [15, 16], such as ESTIMATE (Estimation of Stromal and Immune cells in Malignant Tumor tissues using Expression data), have been developed to predict tumor purity and the infiltration of non-tumor cells by calculating immune and stromal scores by utilizing gene expression data from the Cancer Genome Atlas (TCGA) database [17]. In this ESTIMATE algorithm, we analyzed immune and stromal cells of their specific gene expression characteristics to obtain immune and stromal scores and predict invasion of non-tumor cells [18]. Subsequent researches have shown the effectiveness of applying the ESTIMATE algorithm in various tumors [17, 19-21], although its utility on pancreatic cancer have not been fully revealed.

The present study, for the first time in decades, by utilizing both TCGA pancreatic cancer data and immune scores from ESTIMATE algorithm [15], we obtained a list of genes, non-coding RNAs (ncRNAs) and transcription factors (TFs) that were associated with microenvironment and could predict poor outcomes in pancreatic cancer patients, along with possible effective drugs. Essentially, we have validated the correlation in the International Cancer Genome Consortium (ICGC) database, which contains a different pancreatic cancer cohort.

\section{Methods}

Acquisition of gene expression data of pancreatic cancer from TCGA and ICGC database

Gene expression profile of pancreatic cancer patients and their clinical characteristics were extracted from the TCGA database (https://tcga-data.nci.nih.gov/tcga). Use of the TCGA data adhered to TCGA publication guidelines and policies. By utilizing the ESTIMATE algorithm to the downloaded data, we obtained the immune and stromal scores [22]. In addition, gene expression profiles and clinical data of survival and outcomes for pancreatic cancer patients were extracted from the ICGC database (https://icgc.org) for validation. These normalized data were used for Limma analysis and survival analysis. The summary of the clinical information of pancreatic cancer cohorts from TCGA is presented in Additional file 1: Table S1.

\section{Identification of differentially expressed genes (DEGs) and functional enrichment analysis}

The inclusion criteria for identification of DEGs were set as fold change $(\mathrm{FC})>2$ and adjusted $p$ value $<0.05$. The DEGs were chosen for heatmaps and clustering by means of pheatmap and an open source web tool ClustVis [23]. The KEGG pathway enrichment analysis for identified DEGs was conducted using a web server named KOBAS (version 3.0, KEGG Orthology Based Annotation System) [24] online database server (http://kobas.cbi.pku.edu.cn/) with the thresholds of $p$-value $<0.05$. The flow chart was shown in Fig. 1a.

\section{Construction of PPI network}

STRING (version 10.5) tool was utilized to establish the protein-protein interaction (PPI) network [25]. Subsequently, the visualization and analysis were achieved via Cytoscape (version 3.6.1, http://www.cytoscape.org/) [26]. To locate densely populated regions based on topology, we used the plug-in Molecular COmplex DEtection (MCODE) to filtrate significant modules of the PPI network (the parameters were set to default).

\section{Overall survival curve}

Based on the gene signature of multiple survival-associated DEGs, Kaplan-Meier plots were generated to elaborate the association between patients' 5 -year overall survival and DEGs expression levels. The relationship was tested by log-rank test.

\section{Degree and betweenness analysis of the PPI interaction network with module identification and pivot ncRNA recognition}

The network was analyzed by using Network Analyzer, a Cytoscape plug-in, based on topological parameters 

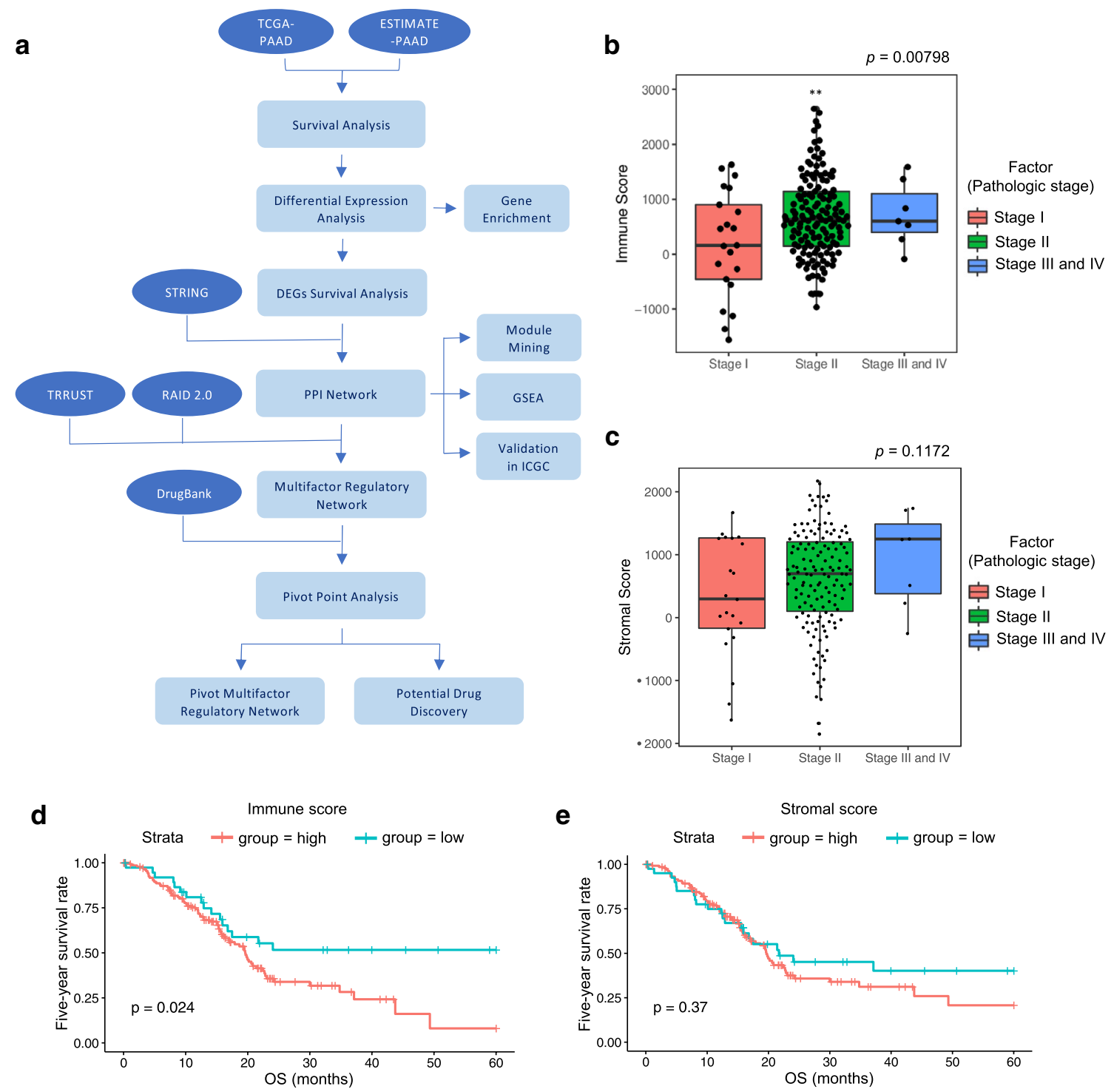

Fig. 1 Immune scores and stromal scores were associated with pathologic stages and overall survival of pancreatic patients. a Work flow of the present study. $\mathbf{b}$ Distribution of immune scores of different pathologic stages. Box-plot showed significant association between pathologic stages and the level of immune scores $(n=177, p=0.00798)$. c Distribution of stromal scores of different pathologic stages. Box-plot showed no significant association between pathologic stages and the level of stromal scores $(n=177, p=0.1172)$. $\mathbf{d}$ TCGA cohort samples were divided into two groups based on their immune scores: the 139 cases with immune scores higher than 0 and the 38 cases with immune scores lower than 0 . As shown in the Kaplan-Meier survival curves, median survival of the low score group was significantly longer than high score group ( $p=0.024$ in log-rank test). e Similarly, TCGA cohort samples were divided into two groups based on their stromal scores: the 135 cases with stromal scores higher than 0 and the 42 cases lower than 0 . The median survival of the low score group was longer than the high score group, althougth not statistically different as indicated by the $p=0.37$ in log-rank test

such as degree and betweenness [27]. In summary, degree illustrated the amount of edges linked to a specific node, while betweenness determined the sum of nonredundant shortest paths passing through a specific node/edge, identifying the network bottleneck [28]. The PPI network was clustered with the ClusterONE algorithms (in Cytoscape) so as to determine functional modules [29].
Based on the PPI network, ClusterONE could detect the protein complex module by altering the most valuable incident and boundary nodes time and again to locally optimize the cohesiveness measure of cluster quality metrics [29]. The pivot ncRNAs that significantly modulate distinct modules and sub-networks were identified by hypergeometric test [30]. 


\section{Analysis of targeting drugs}

The DrugBank online database (https://www.drugbank. $\mathrm{ca} /)$ [31, 32] is contained of biochemical and pharmacological information of any kinds of drugs. In this study, we filtered the data from DrugBank to determine the drug target information of module genes in PPI network and subsequently built drug-target gene interaction networks.

\section{Timer 2.0 database analysis}

To analyze the association of gene expression and immune infiltration levels of immune cells including CD8 + T cell, CD4+ T cell, Treg cell, B cell, neutrophil, macrophage, dendritic cell, natural killer cell and monocyte, the online public resource Tumor Immune Estimation Resource 2.0 (TIMER 2.0; http://timer.cistrome.org/) was utilized [33, 34].

\section{Results}

Immune and stromal scores were associated with pathologic stages and overall survival of pancreatic cancer patients

The gene expression profiles and clinical information of all 177 pancreatic cancer patients (with initial pathologic diagnosis) were downloaded in TCGA PAAD cohort. We included all the pancreatic cancer cases with complete gene expression data and clinical information in the TCGA in our analysis. There clinicopathologic information was listed in Additional file 1: Table S1. According to the clinical information recorded in the TCGA database, we divided all pancreatic cancer cases into Stage I $(21 / 177,11.9 \%)$, Stage II $(146 / 177,82.5 \%)$ and Stage III and IV $(7 / 177,4.0 \%)$ subgroups based on the overall stage of cancer. Their immune and stromal scores were obtained from the ESTIMATE website. The numerical distribution of the two scores was shown in Fig. 1B. In accordance with ESTIMATE algorithm, immune scores were distributed between -1559.87 and 3037.78 and stromal scores ranged from -1843.32 to 2179.19 , respectively. As shown in Fig. 1b, the pancreatic cancer cases at Stage II subgroup had the highest average immune score $(p<0.01)$, followed by Stage III and IV, while the Stage I samples had the lowest immune score. Similarly, the rank order of stromal scores across pancreatic cancer subgroups from highest to lowest was Stage III and
IV $>$ Stage II $>$ Stage I, although the differences were not statistically significant (Fig. 1c), indicating that immune scores were meaningful in the correlation of subgroup classification.

To find out the potential correlation of immune and/or stromal scores with overall survival, we divided the 177 pancreatic cancer cases into high vs. low score groups (using zero as the cut-off point) and performed survival analysis using clinical follow-up data for each set of samples. Kaplan-Meier survival curves indicated that 5-year survival rate of cases with low immune scores was longer (Fig. 1d log-rank $p=0.024$ ). Consistently, although not statistically significant, longer 5 -year overall survival was observed in cases with lower, compared to higher, stromal scores (Fig. 1e, $\log$-rank $p=0.37$ ).

\section{Comparison of gene expression profile with immune and stromal scores in pancreatic cancer}

To explore the correlation of global gene expression profiles with immune and/or stromal scores, we analyzed the RNAseq - HTSeq - FPKM data of all 177 TCGA pancreatic cancer cohorts. As the heatmaps shown in Fig. $2 \mathrm{a}$ and $\mathrm{b}$, there were evident gene expression profiles between cases of high vs. low immune/stromal score groups. According to the immune scores, a total of 2224 DEGs were screened with 1922 upregulated and 302 downregulated genes in high vs. low score groups $(\mathrm{FC}>2$, $p<0.05)$. When it comes to stromal scores, there were 1982 up-regulated genes and 160 down-regulated genes screened in high vs. low score groups $(\mathrm{FC}>2, p<0.05)$. In addition, the Venn diagrams in Fig. 2c, d showed 1611 commonly up-regulated genes and 104 commonly down-regulated genes in the high score groups. There is a certain degree of overlap between the DEGs in highscoring and low-scoring groups of the immune score and the stromal score, especially in the up-regulation group. Among the differentially up-regulated genes, 1611 genes were shared between immune and stromal score groups, accounting for $70.3 \%$ of the genes, while the 104 common down-regulated genes accounted for $29.1 \%$. It is worth mentioning that only immune scores were significantly correlated with 5-year overall survival of patients. Therefore, we were determined to focus on the DEGs from immune score groups for all the following analyses in this manuscript.

\footnotetext{
(See figure on next page.)
}

Fig. 2 Comparison of gene expression profile with immune scores and stromal scores in pancreatic cancer. Heatmaps were drawn based on the methods of average linkage and Pearson distance measurement. Genes with higher expression were shown in red while lower expression genes were in green. a Heatmap of the DEGs of immune scores higher than 0 vs. immune scores lower than $0(p<0.05, F C>2)$. $\mathbf{b}$ Heatmap of the DEGs of stromal scores higher than 0 vs. stromal scores lower than $0(p<0.05, F C>2) . \mathbf{c}$, d Venn diagrams showing the number of commonly upregulated (c) or downregulated (d) DEGs in immune and stromal score groups. Top 10 terms of GO (e), KEGG pathway (f) and KEGG disease (g) were acquired from KOBAS 3.0 annotation and enrichment tool. $p<0.05$ 
a

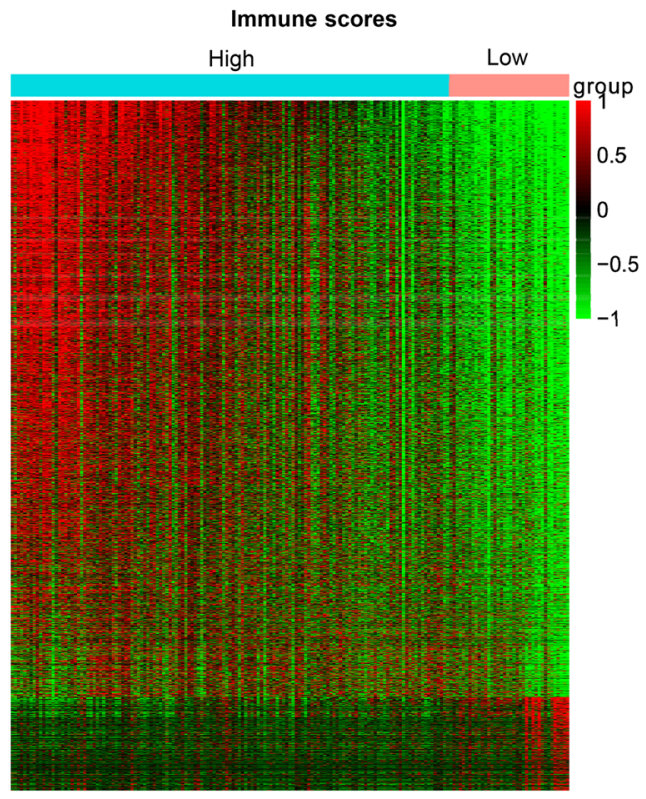

C

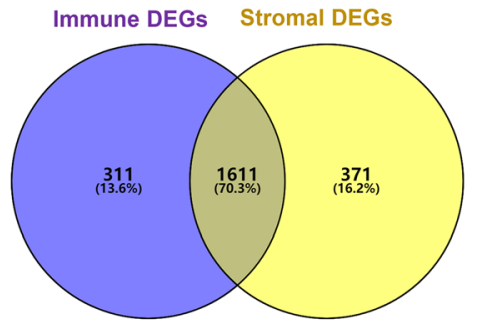

d

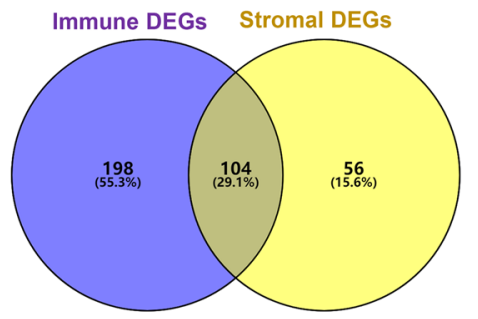

$\mathbf{f}$

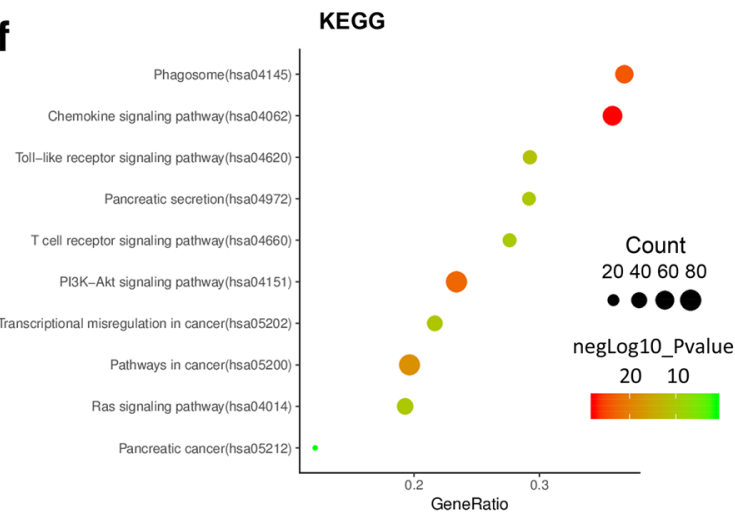

b

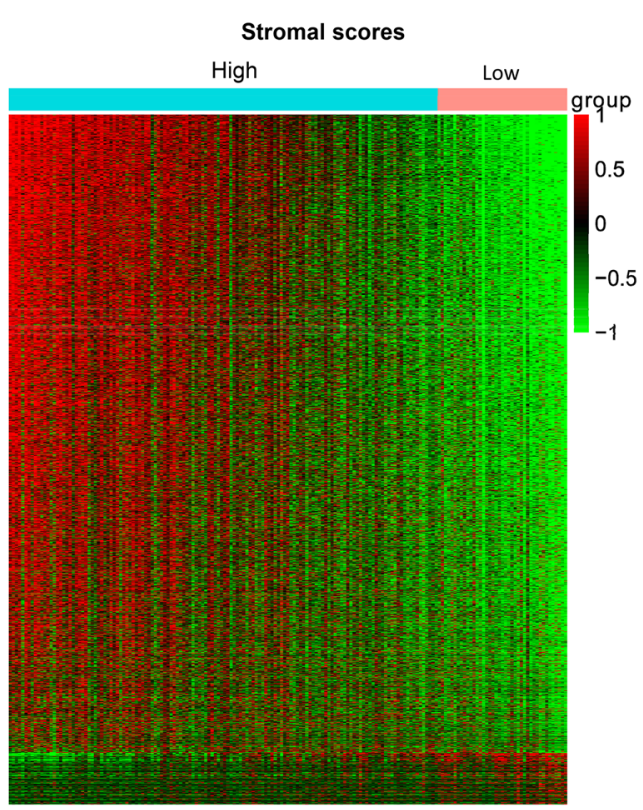

e

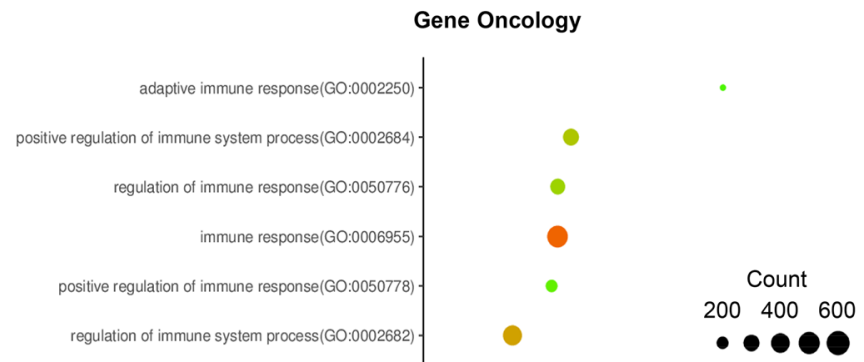

$\bullet \bullet \bullet \bullet \bullet$

negLog10_Pvalue

200100

immune system process(GO:0002376)

immune system development(GO:0002520) -
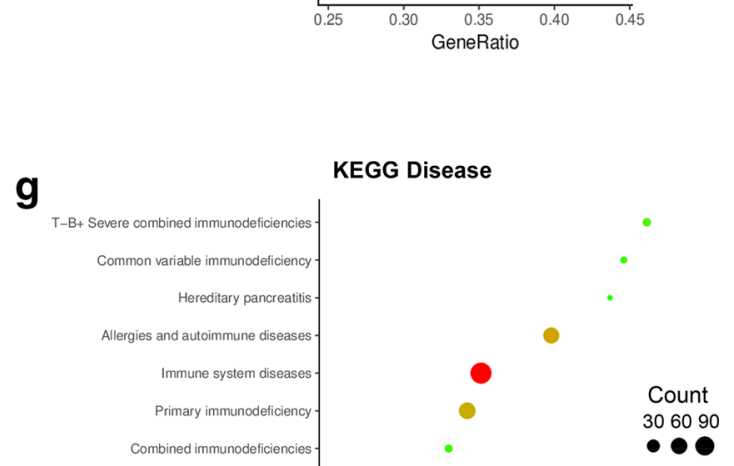

Pancreas diseases - $\quad$ negLog10 Pvalue $\begin{array}{lll}50 & 30 & 10\end{array}$ 
Then, by using KOBAS 3.0, functional enrichment analysis of all upregulated 2224 genes in high-immune score group was carried out in order to sketch the possible effect of the DEGs between the two immune score groups (high and low). The DEGs we obtained in the GO annotation and relevant pathways were enriched in the KEGG database. As shown in Fig. 2e-g, numerous genes were associated with tumor microenvironment or immune function.

\section{Correlation of expression of individual DEGs in overall survival}

To determine the separate effects of the 2224 DEGs on 5-year overall survival, Kaplan-Meier survival curves were generated, of which 246 genes in total were identified to be significantly associated with poor overall survival prediction (log-rank $p<0.05$, Additional file 1 : Figure S1 showed the designated genes).

\section{Protein-protein interactions among genes of prognostic value}

To investigate the interactions among the above DEGs, we utilized the STRING tool and yeasted PPI networks. By limiting the genes with interactions to be only included in these 246 DEGs, we established a PPI network that was significantly associated with 5-year overall survival using Cytoscape software. As suggested by Fig. 3a, 93 nodes and 158 edges composed the network. As former studies reported [35-37] that biological significance exists in PPI networks (nodes=proteins; edges =interactions between proteins), it is generally believed that important proteins often cooperate with many other proteins (such as TFs). In other words, nodes (proteins) with larger node degrees (more interactions and cooperations) in the network are thus more important for further study.

\section{Functional enrichment analysis for DEGs of prognostic value}

To further clarify the main biological functions of the 93 screened DEGs, we performed functional enrichment clustering analysis of the 93 gene nodes using the gene set enrichment analysis (GSEA) method $(p<0.05$, FDR $<0.25)$. As shown in Fig. $3 \mathrm{~b}$ and $\mathrm{c}$, these genes showed strong association with biologically significant processes such as immune response, cell and subcellular composition and movement of cell or subcellular component, which was consistent with the result of PPI network analysis.

\section{Module mining of PPI networks}

To perform the module mining on the previously mentioned PPI networks, we obtained a total of 7 modules using the MCODE tool in Cytoscape. It is worth mentioning that one module contained more than 10 nodes (Fig. 4). CXCL5, which have been reported to recruit and activate leukocytes and play a role in cancer progression, was also indicated to be one of the key genes in the PPI network. The genes in the module, including CXCL9, CXCL10, CXCL11, PPY, LPAR3, HCAR3 and so on, were mainly associated with the prognosis of pancreatic cancer or other tumors as listed according to each node degree in Table 1.

\section{Validation in the ICGC database}

To validate the prognostic significance of the 93 prognosis-related genes identified from the TCGA cohort, we downloaded and analyzed gene expression data and its corresponding clinical information of a cohort of 95 pancreatic cancer cases from ICGC. It showed that this set of data contained 79 of 93 prognostically significant genes and their prognostic values in pancreatic cancer were visualized by Kaplan-Meier survival curves. A total of 26 genes were found to be significantly associated with poor prognosis (Additional file 1: Figure S1), while five of them, including SYT12, GJB5, RHOJ, GJB3 and IFI27, were of particular interest as they have not been previously associated with poor outcomes in pancreatic cancer cases.

\section{Degree and betweenness analysis with prognosis-related DEGs and multi-regulatory network construction}

By using RAID 2.0 and TRRUST v2 database respectively, we investigated the interaction between human ncRNAs along with TFs and the 93 prognosis-related genes. We obtained 8437 out of 1,954,911 pairs of ncRNAs with genes and 179 out of 8427 pairs of TFs with genes. These interaction pairs were imported into Cytoscape software to build a multi-regulatory network with 2228 nodes and

\footnotetext{
(See figure on next page.)

Fig. 3 PPI networks among genes of prognostic value and functional enrichment analysis. a PPI networks among DEGs (93 nodes) that were significantly associated with 5-year overall survival. The color of a node in the network reflected the log (FC) value of the $Z$ score of gene expression. The size of a node indicated the number of interacting proteins with the designated protein. $\mathbf{b}$, c Results of GSEA. GSEA was performed for the 93 DEGs to further screen the significant GO between the higher immune score group and lower immune score group. GSEA, gene set enrichment analysis
} 
a
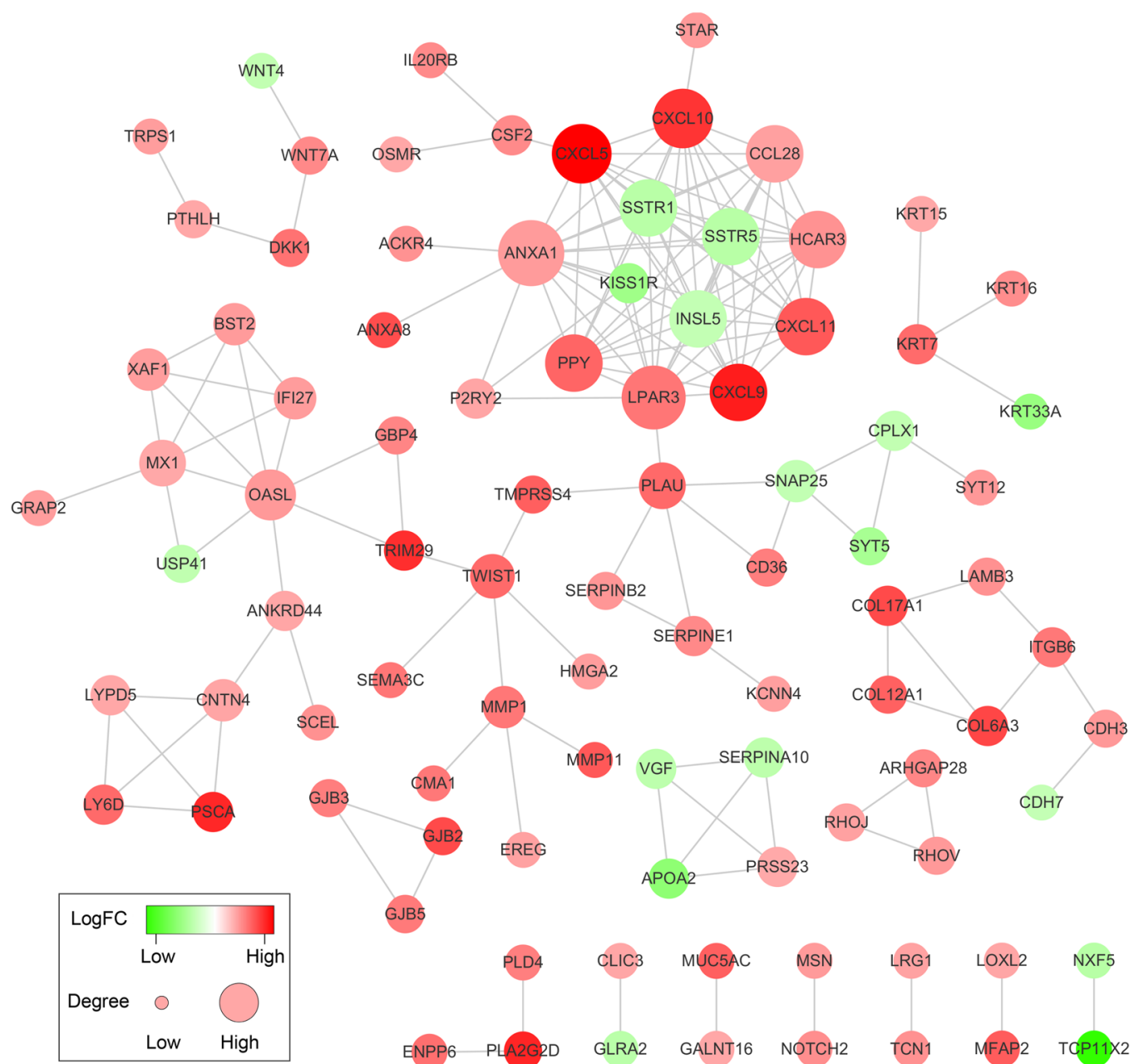

PLD4 CLIC3 MUC5AC MSN LRG1 LOXL2 NXF5

ENPP6 PLA2GRD GLRA2 GALNT16 NOTCH2 TCN1 MFAP2 TCP11X2

b

Enrichment plot: GO - Immune Response

—Enrichment profile — Hits - — Ranking metric scores

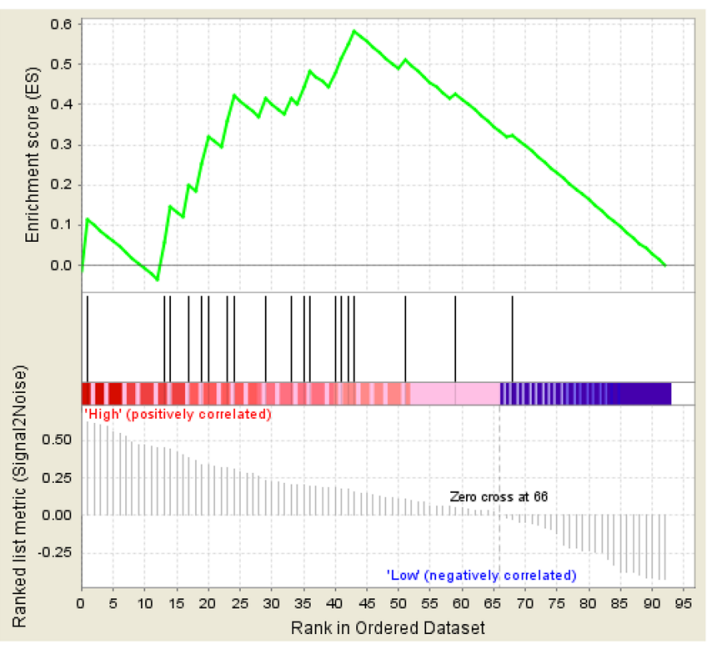

C

Enrichment plot:

GO - Movement of cell OR Subcellular component

- Enrichment profile — Hits - - Ranking metric scores

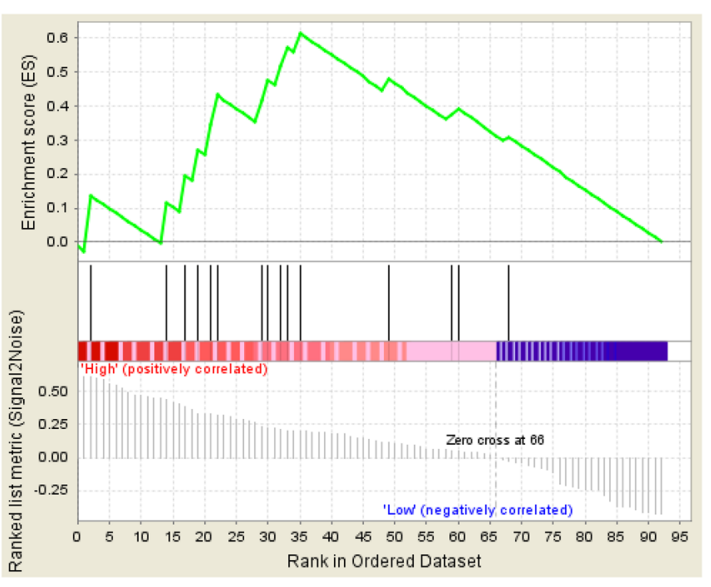




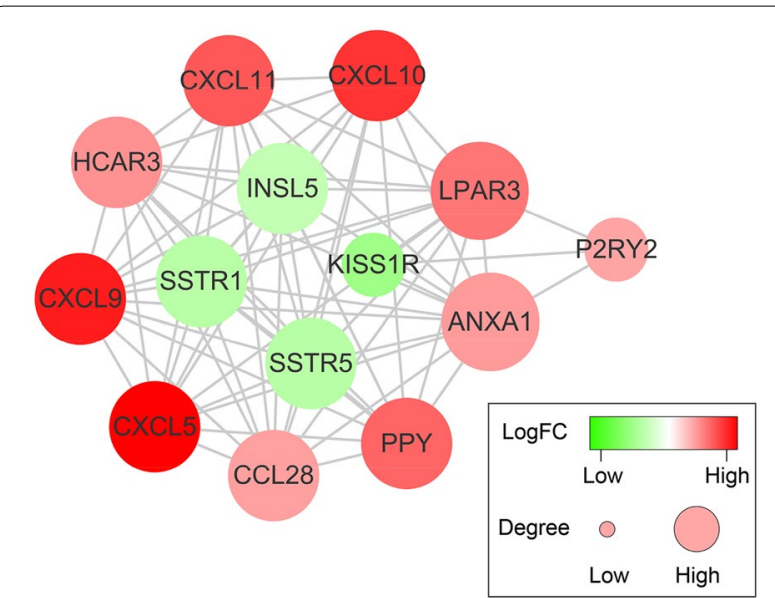

Fig. 4 Module mining of PPI networks. The PPI network data from STRING was further analyzed by Cytoscape. Circles and lines represented genes and the interaction of proteins between genes, respectively. The color of a node in the network reflected the log (FC) value of the $Z$ score of gene expression. The size of a node indicated the number of interacting proteins with the designated protein

Table 1 Module mining results of the genes associated with the prognosis of pancreatic cancer or other cancers

\begin{tabular}{llcl}
\hline Degree & Gene & \multicolumn{1}{l}{ logFC } & Reference \\
\hline 13 & ANXA1 & 1.172288 & PMID:25854353, PMID:27412958 \\
13 & LPAR3 & 1.616316 & PMID:22876164, PMID:26440309 \\
11 & SSTR1 & -1.20245 & PMID:15706439, PMID:18823376 \\
11 & SSTR5 & -1.21848 & PMID:15706439, PMID:26474434 \\
11 & CXCL5 & 2.994452 & PMID:21356384 \\
11 & INSL5 & -1.03296 & PMID:25514935 \\
11 & CXCL10 & 2.382618 & PMID:25415223, PMID:26423423 \\
11 & CXCL11 & 1.970112 & PMID:30747226 \\
11 & CCL28 & 1.114131 & PMID:28092365 \\
11 & CXCL9 & 2.672843 & PMID:30685120, PMID:23394575 \\
11 & HCAR3 & 1.282322 & \\
11 & PPY & 1.816823 & \\
3 & KISS1R & -1.62753 & PMID:23969598, PMID:30123188 \\
3 & P2RY2 & 1.056005 & PMID:30420446 \\
\hline
\end{tabular}

8616 edges, including 2021 miRNA nodes, 3 lncRNA nodes, 89 TF nodes and 91 mRNA nodes (Fig. 5). In order to obtain more accurate and significant results, we used the degree and betweenness analysis method to further screen factors including ncRNAs, TFs and drugs that have significant regulatory effects on prognosisrelated DEGs. Here, a pivotal node was defined as having interactions with at least two prognosis-related genes and the hypergeometric $p$ value was less than 0.05 .
ncRNAs regulate genes associated with significant prognosis

To explore ncRNAs that can significantly regulate prognosis-related mRNAs, we screened the RAID 2.0 database for pivotal ncRNA nodes. By using degree and betweenness analysis, we obtained a total of 42 ncRNA-pivotal nodes, including 39 miRNA nodes and 3 lncRNA nodes. The 10 most hypergeometrically significant ncRNAs, including WSPAR, has-miR-4425, has-miR-4536-5p, has-miR-410-5p, has-miR-8064, has-miR-6871-5p, hasmiR-4767, has-miR-608, has-miR-4481 and has-miR3605-3p, were listed in Additional file 1: Figure S2 (in which the expression of has-miR-4481 was not reported in pancreatic cancer).

TFs regulated genes associated with significant prognosis To explore TFs that can significantly regulate prognosisrelated genes, we screened the TRRUST v2 database for pivotal TF nodes. Accordingly, we put nodes that interacted with at least two prognosis-related genes (hypergeometric $p<0.05)$ into the candidate TF node set. Since we only found one TF, RFWD2, based on the hypergeometric $p$ value, we loosened the $p$ value cut-off to 0.1 and obtained 7 TF-pivotal nodes as listed in Additional file 1: Figure S3. Subsequently, we analyzed the association of the expression level of RFWD2 and immune cell infiltration by utilizing the TIMER 2.0 database (Additional file 1: Figure S4).

\section{Construction of a multi-factor regulatory network based on the pivot nodes}

Next, we pruned the multi-regulatory network constructed above based on the obtained ncRNA nodes and TF nodes and removed the rest of the non-pivot nodes. Since there were four miRNAs, including hsa-miR-3978, hsa-miR-4276, hsa-miR-4481 and hsa-miR-4535, whose expressions were not found in pancreatic cancer, thus, the final network included 119 nodes and 241 edges, including 7 TFs, 3 lncRNAs and 35 miRNAs.

\section{Explore drugs that have a therapeutic effect on pancreatic cancer}

To explore more effective drugs that can significantly regulate prognosis-related genes, we screened the DrugBank database for pivotal interaction nodes. Similarly, a pivotal node was defined as having interactions with at least two prognosis-related genes and the hypergeometric $p$ value was less than 0.05 . A total of 17 drug-pivot nodes were obtained (hypergeometric $p<0.05$ ). The 10 most significant drugs including Amcinonide and Octreotide were listed in Table 2. 
Table 2 Top 10 pivotal drugs that had potential therapeutic effects on pancreatic cancer

\begin{tabular}{ll}
\hline Drug-pivot & $\boldsymbol{p}$ value \\
\hline Fibrinolysin & 0.000526165 \\
Urokinase & 0.00126686 \\
Pasireotide & 0.003062761 \\
Octreotide & 0.003062761 \\
Lutetium Lu 177 dotatate [PMID:22388631, & 0.005028043 \\
PMID:28076709] & \\
Tenecteplase & 0.025271353 \\
Amcinonide & 0.045593349 \\
Botulinum Toxin Type A & 0.045593349 \\
HspE7 [PMID:19225922, PMID:11189443] & 0.045593349 \\
Lanreotide [PMID:26614375, PMID:25060168] & 0.045593349 \\
\hline
\end{tabular}

\section{Discussion}

Recently, increasing evidence has proven that tumor microenvironment plays an essential role in the process of pancreatic cancer [38-41]. Immune cells and stromal cells, the two main types of non-neoplastic components, have been reported to accelerate tumor immune escape and progression [42, 43]. More and more studies have revealed that stroma not only served as a physical barrier to drug delivery and facilitated chemotherapy resistance, but also supported tumor growth and metastasis [44]. Although gemcitabine is presently the standard option in the treatment of pancreatic cancer, stromal components including tumor-associated macrophages have been proven essential in delivering gemcitabine resistance in pancreatic cancer cells [45]. Thus, investigating the mechanism of tumor microenvironment and identifying stromal components could impel a deeper understanding and contribute to a better prognosis of pancreatic cancer patients in clinical practice.

In the present study, we identified numerous tumor microenvironment related DEGs which contributed to pancreatic cancer overall survival in the TCGA database. Especially, by comparing global gene expression in abundant cases with high vs. low immune scores, we obtained 246 genes that significantly correlated with poor overall survival in TCGA pancreatic cancer training cohort. By constructing PPI network and limit the genes only in the 246 DEGs, we obtained 93 gene nodes, in which 79 of them were validated in ICGC pancreatic cancer cohort. The subsequent GSEA method confirmed their strong association with biologically significant processes including immune response and cell and subcellular composition. The module mining further assured their association with the prognosis of pancreatic cancer and beyond. Afterwards, by manipulating RAID 2.0, TRRUST v2 database and pivotal point analysis, we obtained 38 ncRNA-pivotal nodes (with 4 unexpressed miRNAs excluded) and 7 TF-pivotal nodes that interacted with the 93 prognosis-related DEGs. Then, the succeeding multi-factor regulatory network construction was achieved based on the screened pivotal nodes. By utilizing the TIMER 2.0 database, we validated the significant correlation of RFWD2 expression and immune infiltration including CD8+ T cell, CD4+ T cell, B cell, macrophage and natural killer cell. Ultimately, by screening in the DrugBank, we obtained 17 pivotal drugs that were potentially benefit for pancreatic cancer patients.

As illustrated in Fig. 4 and Table 1, of the 14 genes identified, 12 genes (e.g., ANXA1, LPAR3, SSTR1, CXCL5, KISS1R) have been reported to be involved in pancreatic cancer pathogenesis or significant in predicting overall survival, indicating that the big data-based analyses in the current work using TCGA training cohort and ICGC validation cohort have prognostic values. While the remaining two genes, HCAR3 (Hydroxy-Carboxylic Acid Receptor 3) and PPY (Pancreatic Polypeptide), have not previously been linked with pancreatic cancer prognosis yet, this study provides a theoretical basis for investigating their potential role in pancreatic cancer.

Recently, the explosion of studies into ncRNA have provided evidence of their key regulatory roles in shaping oncogenic activity in pancreatic cancer [46-48]. Subsequently, many cancer-focused clinical trials involving ncRNAs as novel biomarkers have begun [49-51]. In our present work, the top 10 ncRNAs with significant association with pancreatic cancer prognosis were listed in Additional file 1: Figure S2, including WSPAR (lncRNA T-Cell Factor-7, LncTCF7), miR-410, miR-608 and so on. For instance, WSPAR had been reported to promote hepatocellular carcinoma aggressiveness through epithelial-mesenchymal transition [52]. It is worth mentioning that the IL- 6 produced by non-neoplastic components within pancreatic tumors could activate proinflammatory STAT3 signaling in hepatocytes [53]. In turn, by enhancing hepatic stellate cell activation, those hepatocytes release myeloid cell chemoattractant proteins to recruit myeloid cells and increase fibrosis deposition [54]. In other words, by means of the secreted IL-6, pancreatic cancer promoted the formation of a pro-metastatic niche in the liver [55]. Thus, it would be meaningful to investigate whether WSPAR was playing essential part in the evolution of pancreatic cancer.

Currently, several clinical trials of adjuvant and neoadjuvant therapies were conducted [56], which are essential for the treatment of pancreatic cancer. As the DrugBank indicated, pivots such as Fibrinolysin and Pasireotide had already been explored in the study of pancreatic cancer $[57,58]$, Amcinonide came to the horizon. Amcinonide as a corticosteroid has been reported to effectively reverse the expression of the oncogene DAPK1 (death-associated 


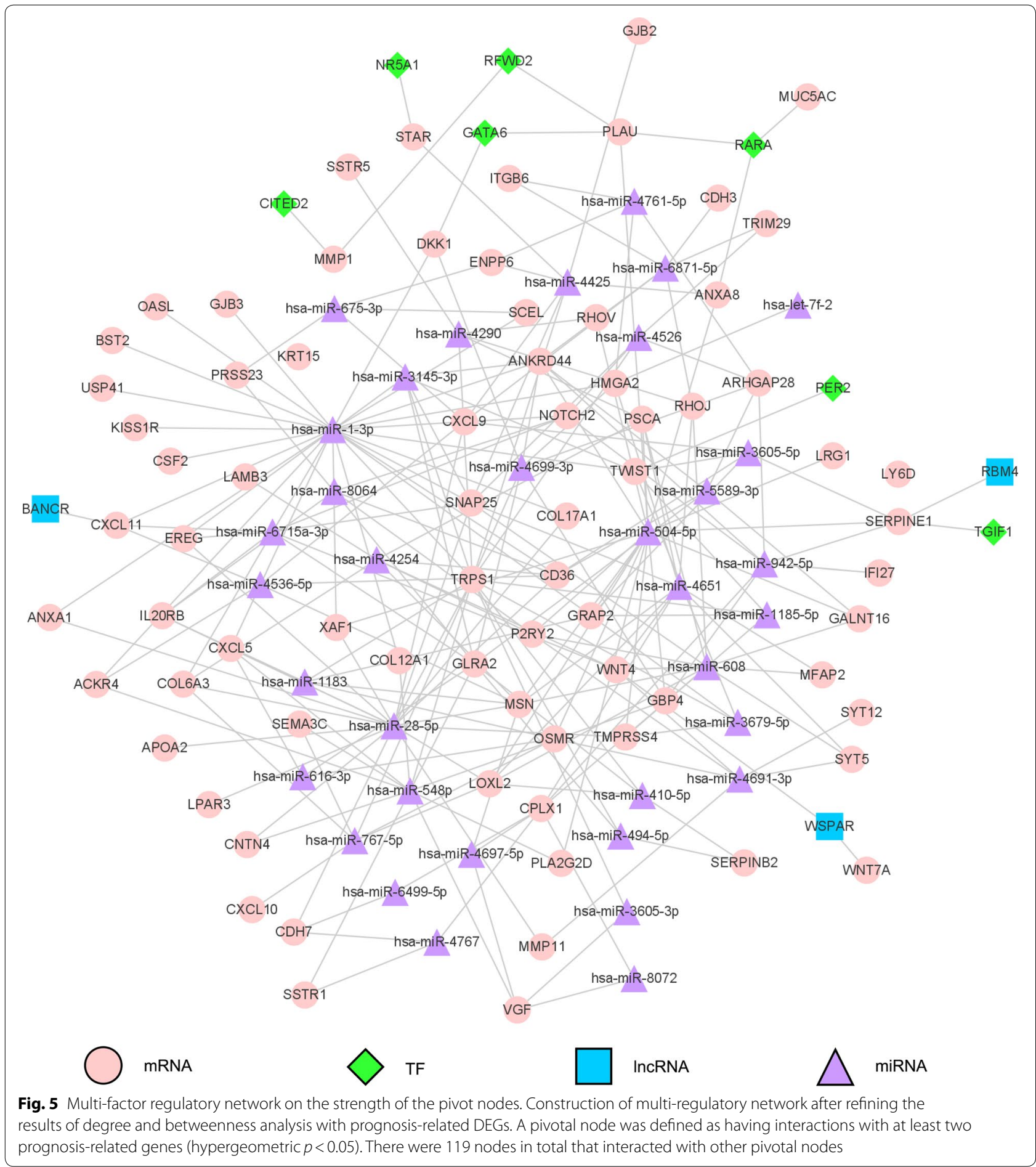

protein kinase 1) in liver carcinoma [59]. While the effect of Amcinonide on pancreatic cancer have not been investigated yet, the present study offered a good insight into the further study of pancreatic cancer.
Pancreatic cancer evolution is essentially affected by the interaction of the intact cancer cells and the microenvironment around them, which then modifies tumor recurrence, drug resistance and prognosis of pancreatic 
cancer [60]. Preceding researches have done active investigation of how the tumor microenvironment was molded by the activated tumor-intrinsic genes [61]. In the present study, we provided a useful further dimension in deciphering the complicated crosstalk between tumor and its microenvironment in pancreatic cancer. Although our study sheds new light on the characteristics of the genes in microenvironment of pancreatic cancer, which in return affected tumor evolution, it still has limitations. The prognosis significance, biological functions and molecular mechanisms of the factors, including HCAR3, PPY, RFWD2, WSPAR and Amcinonide, should be investigated alone and in combination to facilitate the translational research of pancreatic cancer.

\section{Conclusions}

In summary, by means of ESTIMATE algorithm-based immune scoring and functional enrichment analysis of TCGA pancreatic cancer training cohort applied by KOBAS and GSEA, we extracted a list of genes that correlated with tumor microenvironment. These genes were subsequently tested to outline the prognosis of pancreatic cancer in an independent ICGC pancreatic cancer validation cohort. Further investigation of the new targets including DEGs, ncRNAs and TFs lead to novel insights into the potential association of tumor microenvironment with pancreatic cancer prognosis in a comprehensive manner. Drugs with potential therapeutic effects are also worth investigating. Factors such as HCAR3, PPY, RFWD2, WSPAR and Amcinonide appeared above the horizon. Last but not least, despite the grim status quo of pancreatic cancer, we still believe that light is coming [3].

\section{Supplementary information}

Supplementary information accompanies this paper at https://doi. org/10.1186/s12935-020-01426-1.

Additional file 1. Table S1. The clinical information of pancreatic cancer patients in TCGA training cohort. Figure S1. ICGC cohort validation. Figure S2. Top $10 \mathrm{ncRNAs}$ significantly associated with pancreatic cancer prognosis. Figure S3. The pivotal TF nodes obtained by screening the TRRUST v2 database. Figure S4. Correlation of RFWD2 expression with infiltration levels of CD8+ T cell, CD4+ T cell, B cell, macrophage and natural killer cell in pancreatic cancer at TIMER 2.0 database.

\section{Abbreviations}

TCGA: The Cancer Genome Atlas; ESTIMATE: Estimation of Stromal and Immune cells in Malignant Tumor tissues using Expression data; DEG: Differentially expressed gene; ncRNA: Non-coding RNA; ICGC: International Cancer Genome Consortium; KOBAS: KEGG Orthology Based Annotation System; PPI: Protein-protein interaction; MCODE: Molecular Complex Detection; GSEA: Gene set enrichment analysis; TF: Transcription factor; TIMER: Tumor Immune Estimation Resource.

Acknowledgements

Not applicable.

\section{Authors' contributions}

TL performed the data analysis, drafted this manuscript and was a major contributor in writing and revising the manuscript. QiL contributed analysis tools, edited and revised the manuscript. RZ contributed analysis tools. QuL and YZ conceived and designed the study, supervision and funding acquisition. All authors read and approved the final manuscript.

\section{Funding}

This study was supported by the grants from National Natural Science Foundation of China (81673023, 81872501 and 81502068) and Beijing Natural Science Foundation of China (7172177) and Chinese Academy of Medical Sciences \& Peking Union Medical College Postgraduate Innovation Fund Project (2019-1002-14).

\section{Availability of data and materials}

All data generated or analysed during this study are included in this published article.

Ethics approval and consent to participate

Not applicable.

\section{Consent for publication}

Not applicable.

\section{Competing interests}

The authors declare that they have no competing interests.

Received: 25 April 2020 Accepted: 14 July 2020

Published online: 25 July 2020

\section{References}

1. Siegel RL, Miller KD, Jemal A. Cancer statistics, 2019. CA Cancer J Clin. 2019;69(1):7-34.

2. Macedo FI, Ryon E, Maithel SK, Lee RM, Kooby DA, Fields RC, et al. Survival outcomes associated with clinical and pathological response following neoadjuvant FOLFIRINOX or gemcitabine/nab-paclitaxel chemotherapy in resected pancreatic cancer. Ann Surg. 2019;270(3):400-13.

3. Wolfgang CL, Herman JM, Laheru DA, Klein AP, Erdek MA, Fishman EK, et al. Recent progress in pancreatic cancer. CA Cancer J Clin. 2013;63(5):318-48.

4. Conroy T, Desseigne F, Ychou M, Bouche O, Guimbaud R, Becouarn Y, et al. FOLFIRINOX versus gemcitabine for metastatic pancreatic cancer. N Engl J Med. 2011;364(19):1817-25.

5. Binenbaum Y, Na'ara S, Gil Z. Gemcitabine resistance in pancreatic ductal adenocarcinoma. Drug Resist Updat. 2015;23:55-68.

6. Kamisawa T, Wood LD, Itoi T, Takaori K. Pancreatic cancer. Lancet. 2016;388(10039):73-85

7. Feig C, Gopinathan A, Neesse A, Chan DS, Cook N, Tuveson DA. The pancreas cancer microenvironment. Clin Cancer Res. 2012;18(16):4266-76.

8. Sousa CM, Biancur DE, Wang X, Halbrook CJ, Sherman MH, Zhang L, et al. Pancreatic stellate cells support tumour metabolism through autophagic alanine secretion. Nature. 2016;536(7617):479-83.

9. Jiang $\mathrm{H}$, Hegde $\mathrm{S}$, Knolhoff BL, Zhu Y, Herndon JM, Meyer MA, et al. Targeting focal adhesion kinase renders pancreatic cancers responsive to checkpoint immunotherapy. Nat Med. 2016:22(8):851-60.

10. Kalluri R, Zeisberg M. Fibroblasts in cancer. Nat Rev Cancer. 2006;6(5):392-401.

11. Straussman R, Morikawa T, Shee K, Barzily-Rokni M, Qian ZR, Du J, et al Tumour micro-environment elicits innate resistance to RAF inhibitors through HGF secretion. Nature. 2012;487(7408):500-4.

12. Hanahan D, Weinberg RA. Hallmarks of cancer: the next generation. Cell. 2011;144(5):646-74

13. Hanahan D, Coussens LM. Accessories to the crime: functions of cells recruited to the tumor microenvironment. Cancer Cell. 2012;21(3):309-22.

14. Galon J, Pages F, Marincola FM, Thurin M, Trinchieri G, Fox BA, et al. The immune score as a new possible approach for the classification of cancer. J Transl Med. 2012;10:1. 
15. Yoshihara K, Shahmoradgoli M, Martinez E, Vegesna R, Kim H, TorresGarcia W, et al. Inferring tumour purity and stromal and immune cell admixture from expression data. Nat Commun. 2013;4:2612.

16. Carter SL, Cibulskis K, Helman E, McKenna A, Shen H, ZackT, et al. Absolute quantification of somatic DNA alterations in human cancer. Nat Biotechnol. 2012;30(5):413-21.

17. Jia D, Li S, Li D, Xue H, Yang D, Liu Y. Mining TCGA database for genes of prognostic value in glioblastoma microenvironment. Aging (Albany NY). 2018:10(4):592-605.

18. Ni J, Wu Y, Qi F, Li X, Yu S, Liu S, et al. Screening the cancer genome atlas database for genes of prognostic value in acute myeloid leukemia. Front Oncol. 2019;9:1509.

19. Shah N, Wang P, Wongvipat J, Karthaus WR, Abida W, Armenia J, et al. Regulation of the glucocorticoid receptor via a BET-dependent enhancer drives antiandrogen resistance in prostate cancer. Elife. 2017;6:e27861.

20. Priedigkeit N, Watters RJ, Lucas PC, Basudan A, Bhargava R, Horne W, et al. Exome-capture RNA sequencing of decade-old breast cancers and matched decalcified bone metastases. JCI Insight. 2017;2:17.

21. Alonso MH, Ausso S, Lopez-Doriga A, Cordero D, Guino E, Sole X, et al. Comprehensive analysis of copy number aberrations in microsatellite stable colon cancer in view of stromal component. Br J Cancer. 2017;117(3):421-31.

22. Yoshihara K, Shahmoradgoli M, Martínez E, Vegesna R, Kim H, Torres-Garcia W, et al. Inferring tumour purity and stromal and immune cell admixture from expression data. Nat Commun. 2013:4:1-11.

23. Metsalu T, Vilo J. ClustVis: a web tool for visualizing clustering of multivariate data using Principal Component Analysis and heatmap. Nucleic Acids Res. 2015;43(Web Server issue):W566-70.

24. Xie C, Mao X, Huang J, Ding Y, Wu J, Dong S, et al. KOBAS 2.0: a web server for annotation and identification of enriched pathways and diseases. Nucleic Acids Res. 2011;39(Web Server issue):W316-22.

25. Szklarczyk D, Morris JH, Cook H, Kuhn M, Wyder S, Simonovic M, et al. The STRING database in 2017: quality-controlled protein-protein association networks, made broadly accessible. Nucleic Acids Res. 2017;45(Database issue):D362-8

26. Shannon P, Markiel A, Ozier O, Baliga NS, Wang JT, Ramage D, et al. Cytoscape: a software environment for integrated models of biomolecular interaction networks. Genome Res. 2003;13(11):2498-504.

27. Assenov Y, Ramirez F, Schelhorn SE, Lengauer T, Albrecht M. Computing topological parameters of biological networks. Bioinformatics. 2008;24(2):282-4

28. Wu D, Huang Y, Kang J, Li K, Bi X, Zhang T, et al. ncRDeathDB: a comprehensive bioinformatics resource for deciphering network organization of the ncRNA-mediated cell death system. Autophagy. 2015;11(10):1917-26.

29. Nepusz T, Yu H, Paccanaro A. Detecting overlapping protein complexes in protein-protein interaction networks. Nat Methods. 2012;9(5):471-2.

30. Ulitsky I, Shamir R. Pathway redundancy and protein essentiality revealed in the Saccharomyces cerevisiae interaction networks. Mol Syst Biol. 2007;3:104.

31. Wishart DS, Knox C, Guo AC, Shrivastava S, Hassanali M, Stothard P, et al. DrugBank: a comprehensive resource for in silico drug discovery and exploration. Nucleic Acids Res. 2006;34(Database issue):D668-72

32. Wishart DS, Feunang YD, Guo AC, Lo EJ, Marcu A, Grant JR, et al. DrugBank 50: a major update to the DrugBank database for 2018. Nucleic Acids Res. 2018;46(Database issue):D1074-82.

33. LiT, Fu J, Zeng Z, Cohen D, Li J, Chen Q, et al. TIMER20 for analysis of tumorinfiltrating immune cells. Nucleic Acids Res. 2020;48(W1):W509-614.

34. Li B, Severson E, Pignon JC, Zhao H, Li T, Novak J, et al. Comprehensive analyses of tumor immunity: implications for cancer immunotherapy. Genome Biol. 2016;17(1):174.

35. Lu Y, Yang G, Xiao Y, Zhang T, Su F, Chang R, et al. Upregulated cyclins may be novel genes for triple-negative breast cancer based on bioinformatic analysis. Breast Cancer. 2020. https://doi.org/10.1007/s12282-020-01086-z.

36. Dong S, Men W, Yang S, Xu S. Identification of lung adenocarcinoma biomarkers based on bioinformatic analysis and human samples. Oncol Rep. 2020;43(5):1437-50.

37. Ni M, Liu X, Wu J, Zhang D, Tian J, Wang T, et al. Identification of Candidate Biomarkers Correlated With the Pathogenesis and Prognosis of Non-small Cell Lung Cancer via Integrated Bioinformatics Analysis. Front Genet. 2018;9:469.

38. Di Caro G, Cortese N, Castino GF, Grizzi F, Gavazzi F, Ridolfi C, et al. Dual prognostic significance of tumour-associated macrophages in human pancreatic adenocarcinoma treated or untreated with chemotherapy. Gut. 2016;65(10):1710-20.

39. Ligorio M, Sil S, Malagon-Lopez J, Nieman LT, Misale S, Di Pilato M, et al. Stromal microenvironment shapes the intratumoral architecture of pancreatic cancer. Cell. 2019;178(1):160-75.

40. Dominguez CX, Muller S, Keerthivasan S, Koeppen H, Hung J, Gierke S, et al. Single-cell RNA sequencing reveals stromal evolution into LRRC15 + myofibroblasts as a determinant of patient response to cancer immunotherapy. Cancer Discov. 2019;10(2):232-53.

41. Banerjee S, Dudeja V, Saluja A. Unconventional T Cells in the Pancreatic Tumor Microenvironment: thinking Outside the Box. Cancer Discov. 2019;9(9):1164-6.

42. Stromnes IM, DelGiorno KE, Greenberg PD, Hingorani SR. Stromal reengineering to treat pancreas cancer. Carcinogenesis. 2014;35(7):1451-60.

43. Özdemir BC, Pentcheva-Hoang T, Carstens JL, Zheng X, Wu CC, Simpson T, et al. Depletion of carcinoma-associated fibroblasts and fibrosis induces immunosuppression and accelerates pancreas cancer with diminished survival. Cancer Cell. 2014;25(6):719-34.

44. Mahajan UM, Langhoff E, Goni E, Costello E, GreenhalfW, Halloran C, et al. Immune cell and stromal signature associated with progression-free survival of patients with resected pancreatic ductal adenocarcinoma. Gastroenterology. 2018;155(5):1625.

45. Halbrook CJ, Pontious C, Kovalenko I, Lapienyte L, Dreyer S, Lee HJ, et al. Macrophage-Released Pyrimidines Inhibit Gemcitabine Therapy in Pancreatic Cancer. Cell Metab. 2019;29(6):1390.

46. Yang Y, Ishak Gabra MB, Hanse EA, Lowman XH, Tran TQ, Li H, et al. MiR-135 suppresses glycolysis and promotes pancreatic cancer cell adaptation to metabolic stress by targeting phosphofructokinase-1. Nat Commun. 2019:10(1):809.

47. Zhang J, Bai R, Li M, Ye H, Wu C, Wang C, et al. Excessive miR-25-3p maturation via N(6)-methyladenosine stimulated by cigarette smoke promotes pancreatic cancer progression. Nat Commun. 2019;10(1):1858.

48. Fesler A, Ju J. Development of microRNA-based therapy for pancreatic cancer. J Pancreatol. 2019;2(4):147-51.

49. Rupaimoole R, Slack FJ. MicroRNA therapeutics: towards a new era for the management of cancer and other diseases. Nat Rev Drug Discov. 2017;16(3):203-22.

50. Wang P, Zhang J, Zhang L, Zhu Z, Fan J, Chen L, et al. MicroRNA 23b regulates autophagy associated with radioresistance of pancreatic cancer cells. Gastroenterology. 2013;145(5):1133.

51. Wang P, Zhang L, Chen Z, Meng Z. MicroRNA targets autophagy in pancreatic cancer cells during cancer therapy. Autophagy. 2013;9(12):2171-2.

52. Wu J, Zhang J, Shen B, Yin K, Xu J, Gao W, et al. Long noncoding RNA IncTCF7, induced by IL-6/STAT3 transactivation, promotes hepatocellular carcinoma aggressiveness through epithelial-mesenchymal transition. J Exp Clin Cancer Res. 2015;34:116

53. Razidlo GL, Burton KM, McNiven MA. Interleukin-6 promotes pancreatic cancer cell migration by rapidly activating the small GTPase CDC42. J Biol Chem. 2018:293(28):11143-53.

54. Houg DS, Bijlsma MF. The hepatic pre-metastatic niche in pancreatic ductal adenocarcinoma. Mol Cancer. 2018;17:1-8.

55. Huang L, Hu B, Ni J, Wu J, Jiang W, Chen C, et al. Transcriptional repression of SOCS3 mediated by IL-6/STAT3 signaling via DNMT1 promotes pancreatic cancer growth and metastasis. J Exp Clin Cancer Res. 2016;35:27.

56. Maeda S, Unno M, Yu J. Adjuvant and neoadjuvant therapy for pancreatic cancer. Journal of Pancreatology. 2019;2:3.

57. Zheng D, Chen H, Bartee MY, Williams J, Davids JA, Lomas DA, et al. Myxomaviral anti-inflammatory serpin reduces myeloid-derived suppressor cells and human pancreatic cancer cell growth in mice. J Cancer Sci Ther. 2013;5:291-9.

58. Suleiman Y, Mahipal A, Shibata D, Siegel EM, Jump H, Fulp WJ, et al. Phase I study of combination of pasireotide LAR + gemcitabine in locally advanced or metastatic pancreatic cancer. Cancer Chemother Pharmacol. 2015;76(3):481-7.

59. Li L, Guo L, Wang Q, Liu X, Zeng Y, Wen Q, et al. DAPK1 as an independent prognostic marker in liver cancer. PeerJ. 2017;5:e3568.

60. Ying H, Elpek KG, Vinjamoori A, Zimmerman SM, Chu GC, Yan H, et al. Pten is a major tumor suppressor in pancreatic ductal adenocarcinoma and regulates an NF-kB-cytokine network. Cancer Discov. 2011;1(2):158-69. 
61. Ying H, Dey P, Yao W, Kimmelman AC, Draetta GF, Maitra A, et al. Genetics and biology of pancreatic ductal adenocarcinoma. Genes Dev. 2016;30(4):355-85.

\section{Publisher's Note}

Springer Nature remains neutral with regard to jurisdictional claims in published maps and institutional affiliations.
Ready to submit your research? Choose BMC and benefit from:

- fast, convenient online submission

- thorough peer review by experienced researchers in your field

- rapid publication on acceptance

- support for research data, including large and complex data types

- gold Open Access which fosters wider collaboration and increased citations

- maximum visibility for your research: over $100 \mathrm{M}$ website views per year

At BMC, research is always in progress.

Learn more biomedcentral.com/submissions 